\title{
Estudo da carga de doença das condições orais em Minas Gerais, Brasil, 2004-2006
}

\author{
A study of the overall burden of oral disease \\ in the state of Minas Gerais, Brazil: 2004-2006
}

${ }^{1}$ Departamento de Epidemiologia e Métodos Quantitativos em Saúde, Escola Nacional de Saúd Pública Sergio Arouca,

Fundação Instituto Oswald Cruz. R. Leopoldo Bulhões 1480/814, Manguinhos. 21.041-210 Rio de Janeiro RJ Brasil. correamota@gmail.com
Abstract Oral disease is a major public health problem due to its prevalence in practically all age groups. This is a study of the overall burden of dental caries, edentulism and periodontal disease. The Disability-Adjusted Life Year (DALY) indicator was used as it simultaneously measures the impact of mortality and of health problems that affect the quality of life. The overall burden of oral diseases was analyzed in absolute terms and in rates per 1000 inhabitants. The number of Years Lived with Disability (YLD) due to dental caries was 4,489. An estimated 33,888 YLD were lost due to edentulism, with a rate of 1.8/1000 (2.2/ 1000 among women). An estimated 3217 YLD were lost due to periodontal disease, with a rate of $0.2 / 1000$ for both sexes. Edentulism was the condition that contributed most to the overall burden of oral diseases and is more frequent among women. Higher rates of edentulism and periodontal disease were found in persons aged 45-69 years, while dental caries was more frequent from 1549 years. The overall burden of oral disease studies represent an important aspect of health diagnosis, integrating both fatal and non-fatal outcomes. Furthermore, these studies provide highly relevant information for preventive and therapeutic policy making.

Key words Disability-Adjusted Life Years, Epidemiology, Oral health
Resumo Os agravos orais são um problema de saúde pública devido à sua prevalência em praticamente todos os grupos etários. Estudo da Carga Global de Doença para cárie dentária, edentulismo e doença periodontal. Utilizou-se o DALY, que mede simultaneamente o impacto da mortalidade e dos problemas de saúde que afetam a qualidade de vida. A Carga de Doença para condições orais foi analisada em números absolutos e taxas $/ 1.000$ habitantes. O número de YLD para cárie foi de 4.489. Estimou-se 33.888 YLD para edentulismo, com taxa de 2,2/1.000 nas mulheres. Na doença periodontal estimou-se 3.217 YLD, resultando em uma taxa de 0,2/1.000 para ambos os sexos. $O$ edentulismo contribuiu mais para a carga de doença orais, sendo mais frequente nas mulheres. Maiores taxas de edentulismo e doença periodontal foram de 45-69 anos, enquanto cárie de 15-49 anos. Os estudos de Carga Global de Doença representam um importante aspecto do diagnóstico de saúde, integrando desfechos fatais e não fatais. Além disso, disponibiliza informações de grande relevância na formulação de políticas de prevenção e tratamento.

Palavras-chave Anos de vida perdidos ajustados por incapacidade, Epidemiologia, Saúde bucal 


\section{Introdução}

Os agravos orais são um problema de saúde pública de relevante importância devido a sua significativa prevalência em praticamente todos os grupos etários ${ }^{1}$. A cárie dentária e a doença periodontal são causas de perda dentária que podem gerar edentulismo ${ }^{2}$. As consequências dos agravos orais envolvem aspectos relacionados à comunicação, à funcionalidade da capacidade mastigatória, bem como à autoimagem ${ }^{3}$.

Culturalmente, a dentição sempre esteve associada à idade, vinculando-se a ausência de dentes ao surgimento da velhice. Até meados do século 20 , a grande maioria dos idosos não tinha dentes, mas, nas últimas décadas, a busca por tratamento odontológico, principalmente nos países industrializados, gerou redução da prevalência de dentes perdidos, modificando a relação entre idade avançada e edentulismo ${ }^{4}$.

Tanto em regiões desenvolvidas como em desenvolvimento a tendência foi de queda na prevalência de cárie dentária e edentulismo nas últimas décadas ${ }^{5,6}$, ainda que tenham sido observadas diferenças na distribuição de casos entre diferentes grupos socioeconômicos, caracterizando persistentes desigualdades em saúde ${ }^{7-9}$.

No Brasil, evidencia-se uma melhora na saúde bucal em anos mais recentes. Em 1986, o índice CPOD (número de dentes cariados, perdidos e obturados) esteve entre os maiores do mundo, em todas as faixas etárias, alcançando um valor médio de 6,6 dentes para crianças de 12 anos. Após dezessete anos, em 2003, este índice apresentou redução para 2,8 dentes por pessoa. Entretanto, o percentual de indivíduos com periodonto saudável ainda apresentava valores baixos, chegando a $46,0 \%$ em crianças, $22,0 \% \mathrm{em}$ adultos e $8 \%$ em idosos ${ }^{10}$.

A mensuração das condições orais pode ser também empiricamente investigada por meio de medidas sintéticas. Uma delas é o indicador composto que mede, simultâneamente, o impacto da mortalidade e dos problemas de saúde na população e quantifica a carga de determinada doença, denominado DALY (Disability Adjusted Life Year). DALY para uma doença ou conjunto de doenças é a soma dos anos de vida perdidos devido à morte prematura (Years of Life Lost) e anos de vida vividos com incapacidade (Years Lived With Disability). Um DALY pode ser interpretado como um ano de vida saudável perdido devido ao agravo ${ }^{11}$.

No mundo, estimou-se que, em 2004, a taxa de anos de vida perdidos ajustados por incapaci- dade (DALY) devido às condições orais foi de 1,2 por 1.000 habitantes ${ }^{11}$. O primeiro estudo de Carga Global de Doença realizado no Brasil, em 1998, estimou uma taxa de 3,0 por 1.000 habitantes, representando $1,2 \%$ do total da carga global de doença do país ${ }^{12}$.

Os agravos orais e suas consequencias perfazem um grupo de doenças que atualmente recebe prioridade dos programas de saúde nacionais. Como estão associados a algumas doenças cardiovasculares, câncer, doenças respiratórias e diabetes, além de serem fortemente influenciadas por fatores comportamentais, as estimativas de morbidade supracitadas justificam sua importância no contexto das políticas de saúde.

O objetivo deste estudo foi apresentar a carga global das doenças orais, no período de 2004 a 2006, para as Macrorregionais de Saúde de Minas Gerais, incluindo os métodos e processos usados nas estimações e discutir vantagens e limitações dos mesmos.

\section{Métodos}

Estudo descritivo da carga global de doença das condições orais em Minas Gerais e suas Macrorregionais de Saúde, no período de 2004 a 2006, por sexo, faixa etária. Os agravos contemplados foram cárie dentária, doença periodontal e edentulismo.

$\mathrm{O}$ indicador usado para mensurar a carga global de doença foi o DALY (Disability Adjusted Life Years - anos de vida perdidos ajustados por incapacidade), medida que estende o conceito de anos potenciais de vida perdidos por morte prematura ao contemplar anos equivalentes de vida saudáveis perdidos devido a problemas de saúde ou incapacidade. O DALY constitui-se na soma de dois componentes: anos de vida perdidos por morte prematura (Years of Life Lost, YLL) e anos de vida vividos com incapacidade (Years Lived With Disability, YLD $)^{11}$.

\section{Cálculo do componente de mortalidade - YLL}

O componente que mede o efeito da mortalidade no DALY, o número de anos de vida perdidos devido à morte prematura para um indivíduo com idade $x$ é, basicamente, a expectativa de vida observada àquela idade. No cálculo do YLL foi utilizado o Sistema de Informações de Mortalidade (SIM) do Ministério da Saúde, referente ao período 2004 a 2006, considerando uma cor- 
reção de sub-registro por sexo e faixa etária e redistribuição dos óbitos por causas mal definidas ${ }^{13}$. Após as estimativas para o cômputo dos três agravos orais observou-se não haver, no período analisado, óbitos tendo como causas básicas essas condições, sendo o total da carga global de doenças desses agravos atribuído ao componente de morbidade YLD.

\section{Cálculo do componente de morbidade - YLD}

$\mathrm{Na}$ estimação do YLD, utilizaram-se, como parâmetros clínico-epidemiológicos, os casos incidentes, a duração e os pesos das incapacidades. Os pesos permitem a soma dos componentes YLL e YLD e são medidas representativas do declínio no estado de saúde, cuja escala varia de zero (saúde plena) a 1 (saúde equivalente à morte $)^{13}$.

No caso dos agravos orais contemplados neste estudo, foi necessário estimar as incidências de cárie dentária, doença periodontal e edentulismo por sexo e faixa etária, informações menos disponíveis do que prevalência. Não foram encontrados estudos que apresentassem estimativas de incidência para cárie dentária, doença periodontal e edentulismo para o Estado de Minas Gerais no período 2004 -2006 e os processos para obtenção dos casos incidentes de cada agravo oral estão descritos a seguir.

\section{Estimativa de incidência de cárie dentária}

Ainda que a cárie dentária seja usualmente medida por meio dos índices CPOD e CEO, os estudos de Carga Global de Doença consideram a mensuração do agravo por pessoa e não por número de dentes, razão pela qual estes índices não foram considerados neste estudo. Desta forma, esta condição oral, assim como nos estudos da Carga Global de Doença, foi definida como episódios de cárie dentária por pessoa, calculando-se incidências para duas sequelas destes episódios: casos que receberam tratamento, considerados como dentes obturados, e casos sem tratamento, considerados como dentes perdidos.

A incidência foi obtida do inquérito Pesquisa Mundial de Saúde Atenção Básica (PMS AB) a partir da assistência recebida no ano anterior à pesquisa. Este inquérito, realizado em 2005, objetivou desenvolver indicadores para avaliação da atenção básica, contemplando também a atenção bucal ${ }^{14}$. Os indivíduos que receberam tratamento dental foram classificados como casos tratados e aqueles que receberam assistência odontológica relativa à extração dentária foram classificados na sequela de casos não tratados. Por último, indivíduos que receberam os demais tratamentos odontológicos foram classificados como "outros tratamentos". Para aqueles que responderam terem realizado, simultaneamente, tratamento dental e extração de dente alocou-se para tratamento dental, considerado como de menor gravidade.

A cárie dentária foi considerada como dependente de um modelo de regressão multinomial que incluiu, como fatores independentes de nível individual, a faixa etária e, como fatores contextuais, o PIB per capita e a proporção de indivíduos com menos de oito anos de escolaridade, dentre os indivíduos com 25 anos e mais.

Com base nos coeficientes do modelo gerado, foi aplicada uma função logística para estimar, por sexo e faixa etária, a incidência de cárie para o Estado de Minas Gerais e Macrorregionais de Saúde, tomando-se as informações de PIB per capita e proporção de indivíduos com menos de oito anos de estudo de cada localidade.

Entretanto, como a PMS AB foi conduzida em indivíduos de 18 anos e mais de idade, não foi possível extrair deste inquérito a incidência para faixas etárias mais jovens. A incidência de obturação e perda dentária por cárie para menores de um ano foi assumida como zero. Para as faixas etárias de 1 a 6 anos e 7 a 14 anos, as estimativas consistiram em uma interpolação por idade, utilizando-se a distribuição proporcional de dentes perdidos e obturados das faixas etárias-índice de 5 e 12 anos da Pesquisa Nacional de Saúde Bucal de $2003^{15}$.

Quanto ao peso da incapacidade, assumiuse um valor de 0,005 para casos de obturação e 0,014 para casos de perda ${ }^{16}$. Uma vez que não foram localizados estudos nacionais que disponibilizassem a duração dos episódios de cárie dentária que resultam em obturação ou perda, considerou-se o consenso do Australian Institute of Health and Welfare ${ }^{17}$ para o qual a duração da doença, para ambos os sexos, é de, em média, 2,5 anos para cárie dentária tratada com obturação e 2 anos para cárie dentária não tratada que resulta em perda de dente.

\section{Estimativa de incidência \\ de doença periodontal}

A doença periodontal foi definida como a presença de bolsa profunda ( $6 \mathrm{~mm}$ ou mais), atendendo a proposta do Estudo da Carga Global de Doençą ${ }^{16}$ e as informações são provenien- 
tes do inquérito Pesquisa Nacional de Saúde Bucal, de $2003^{15}$. A partir das prevalências estimadas para as faixas etárias de 35 a 44 anos e de 65 a 74 anos deste inquérito, projeções foram calculadas para as faixas etárias do presente estudo.

Foram igualmente calculadas a variação das idades, que se constituíram na diferença (em anos) dos pontos médios de classe das faixas etárias de 35-44 anos e 65-74 anos, e a variação da proporção de bolsa profunda, que resultou na diferença entre a proporção de bolsa profunda nas faixas etárias de 35-44 anos e 65-74 anos. Para cada faixa etária a ser projetada havia um ponto médio de classe. O cálculo da proporção de bolsa profunda estimada para determinada faixa etária resultou na multiplicação da razão entre as variações de proporção e idade pela diferença dos pontos médios de classe entre esta faixa etária e a imediatamente anterior.

As informações de prevalência foram utilizadas para se obter os casos incidentes por meio do software DISMOD II, assim como a duração do agravo. Este programa, de domínio livre, é usado para estimar parâmetros necessários para o cálculo do YLD, tais como incidência e duração do agravo, além de aumentar a consistência das informações disponíveis ${ }^{18}$. O peso da incapacidade foi de 0,001 tanto para tratados quanto não tratados ${ }^{19}$ e a duração da doença foi fornecida pelo output do DISMOD II.

\section{Estimativas de incidência de edentulismo}

A definição de edentulismo utilizada no presente estudo foi a ausência de todos os dentes naturais, conceito empregado nos estudos de Carga Global de Doençą ${ }^{16}$.

A partir do inquérito PMS AB, o edentulismo foi definido como uma variável dicotômica que assumiu os valores 1 (edêntulo) e zero (não edêntulo). Um modelo de regressão logística foi usado tendo como variável dependente o edentulismo e como variáveis explicativas, em nível individual, o sexo e a faixa etária. O PIB per capita e a proporção de indivíduos com menos de oito anos de escolaridade, dentre os indivíduos com 25 anos de idade ou mais, foram considerados como variáveis contextuais.

Com base nos coeficientes do modelo gerado, foi aplicada uma função logística para estimar, por sexo e faixa etária, as prevalências de edentulismo para Minas Gerais e Macrorregiões de Saúde, tomando-se as informações de PIB per capita e proporção de indivíduos com menos de oito anos de estudo de cada localidade.
Os casos incidentes, por sexo e faixa etária, e a duração foram obtidos a partir dos casos prevalentes, através de modelagem com o software DISMOD II.

O peso da incapacidade utilizado foi obtido através de média ponderada, no qual o peso de 0,001 referiu-se aos casos tratados, assumindose que $70 \%$ da população brasileira é tratada para edentulismo no Brasil e um peso de 0,061 aos não tratados ${ }^{19}$ conforme estudo da Carga Global de Doença para a região da América Latina e Caribe, obtendo-se um valor de 0,019. A estimativa de duração usada foi a fornecida pelo software DISMOD II que calculou como sendo a própria expectativa de vida.

\section{Análise}

As prevalências, as incidências e os anos de vida vividos com incapacidade (YLD) de cárie dentária, doença periodontal e edentulismo foram analisadas em termos de taxas por 100.000 habitantes, por sexo e faixa etária. Analisaram-se também os anos de vida vividos com incapacidade (YLD) por meio de distribuição proporcional com os grandes grupos de doenças infecciosas e parasitárias, doenças maternas, perinatais e nutricionais (Grupo I), doenças não transmissíveis (Grupo II) e causas externas (Grupo III).

As taxas do YLD (por 100.000 habitantes) de cárie dentária, doença periodontal e edentulismo para as Macrorregionais de Saúde de Minas Gerais e para cada sexo, foram padronizadas por faixa etária segundo a população de Minas Gerais por sexo. Razões de taxas foram obtidas tomando-se a de Minas Gerais como referência.

A comparação da prevalência de edentulismo por Macrorregionais de Saúde e sexo considerou as faixas etárias dos indivíduos de 45 anos e mais e naqueles de 60 anos e mais. Esta análise foi conduzida somente para o edentulismo, uma vez que este agravo foi o único que apresentou diferenças expressivas entre sexos.

\section{Resultados}

A Tabela 1 mostra as taxas de prevalências e de incidência de cárie dentária, doença periodontal e edentulismo por sexo e faixa etária. No caso da cárie dentária, as taxas de incidência de obturação expressaram redução a partir da faixa etária de 7 a 14 anos, ao passo que as taxas de perda dentária expressaram redução somente a partir de 60 anos. A taxa de incidência de obturação 
Tabela 1. Distribuição das taxas* de prevalência e incidência de cárie dentária, edentulismo e doença periodontal, por faixa etária e sexo. Minas Gerais, 2004-2006.

\begin{tabular}{|c|c|c|c|c|c|c|}
\hline \multirow[b]{2}{*}{$\begin{array}{l}\text { Sexo/Faixa } \\
\text { Etária }\end{array}$} & \multicolumn{2}{|c|}{ Cárie } & \multicolumn{2}{|c|}{ Doença Periodontal } & \multicolumn{2}{|c|}{ Edentulismo } \\
\hline & $\begin{array}{c}\text { Taxa de } \\
\text { Incidência } \\
\text { Obturação }\end{array}$ & $\begin{array}{c}\text { Taxa de } \\
\text { Incidência } \\
\text { Perda }\end{array}$ & $\begin{array}{c}\text { Taxa de } \\
\text { Prevalência }\end{array}$ & $\begin{array}{c}\text { Taxa de } \\
\text { Incidência }\end{array}$ & $\begin{array}{c}\text { Taxa de } \\
\text { Prevalência }\end{array}$ & $\begin{array}{c}\text { Taxa de } \\
\text { Incidência }\end{array}$ \\
\hline \multicolumn{7}{|l|}{ Masculino } \\
\hline$<1$ & 0 & 0 & 0 & 0 & 0 & 0 \\
\hline $1-6$ & 4.825 & 156 & 0 & 0 & 0 & 0 \\
\hline $7-14$ & 21.342 & 467 & 0 & 0 & 0 & 0 \\
\hline $15-29$ & 18.792 & 4.969 & 8.021 & 5.495 & 337 & 48 \\
\hline $30-44$ & 18.260 & 5.477 & 17.379 & 9.930 & 1.734 & 143 \\
\hline $45-59$ & 13.319 & 6.309 & 24.531 & 12.628 & 11.454 & 1.417 \\
\hline $60-69$ & 4.273 & 1.613 & 35.815 & 16.546 & 35.862 & 4.506 \\
\hline $70-79$ & 2.290 & 864 & 38.419 & 14.967 & 50.154 & 101 \\
\hline $80+$ & 929 & 351 & 34.793 & 11.899 & 50.436 & 0 \\
\hline \multicolumn{7}{|l|}{ Feminino } \\
\hline$<1$ & 0 & 0 & 0 & 0 & 0 & 0 \\
\hline $1-6$ & 4.825 & 156 & 0 & 0 & 0 & 0 \\
\hline $7-14$ & 21.342 & 467 & 0 & 0 & 0 & 0 \\
\hline $15-29$ & 18.792 & 4.969 & 8.114 & 5.547 & 678 & 96 \\
\hline $30-44$ & 18.260 & 5.477 & 17.387 & 9.933 & 3.323 & 269 \\
\hline $45-59$ & 13.319 & 6.309 & 24.572 & 12.649 & 18.007 & 2.192 \\
\hline $60-69$ & 4.273 & 1.613 & 35.869 & 16.545 & 46.172 & 5.212 \\
\hline $70-79$ & 2.290 & 864 & 38.373 & 14.929 & 59.749 & 116 \\
\hline $80+$ & 929 & 351 & 34.771 & 11.893 & 60.007 & 0 \\
\hline
\end{tabular}

"taxa por 100.000 habitantes

Fonte: Núcleo de Pesquisa em Métodos Aplicados aos Estudos de Carga Global de Doença, Escola Nacional de Saúde Pública Sergio Arouca, Fundação Oswaldo Cruz.

nos indivíduos de 1 a 6 anos foi de 4.825/100.000 habitantes-ano (para ambos os sexos), ao passo que nos idosos de 80 anos e mais declinou para 929/100.000 habitantes-ano. Os casos novos de perda dentária por cárie deteve aumento até a faixa etária de 45 a 59 anos, alcançando 6.309 casos por 100.000 habitantes-ano, declinando a partir de 60 anos e mais.

No que tange à doença periodontal, as prevalências apresentaram tendências crescentes por idade até a faixa etária de 70 a 79 anos $(38.419$ e 38.373/100.000 habitantes para homens e mulheres, respectivamente), quando, a partir de 80 anos, a propensão é de queda nas proporções de indivíduos atingidos. As taxas de incidência deste agravo apresentaram comportamento semelhante às prevalências, ou seja, houve gradual aumento, por faixa etária, nas taxas de incidência. Também a partir de 70 anos, o número de indivíduos que se tornaram doentes sofre redução, que se reflete em taxas menores para ambos os sexos.

De forma geral, o diferencial por sexo apresentou-se somente para o edentulismo, no qual as prevalências e incidências do sexo feminino fo- ram superiores para todas as faixas etárias analisadas. As taxas de prevalência deste agravo foram consideravelmente crescentes até a faixa etária de 70 a 79 anos, alcançando 50.154/100.000 habitantes no sexo masculino e 59.749/100.000 habitantes no feminino. No tocante às taxas de incidência, foram observados valores crescentes até a faixa etária de 60 a 69 anos em ambos os sexos e decréscimo de casos novos, por ano, nas faixas etárias posteriores (70 a 79 anos e 80 e mais).

$O$ diferencial nas prevalências de edentulismo entre os indivíduos de 45 anos ou mais e de 60 anos ou mais, segundo as Macrorregionais de Saúde de Minas Gerais, estão representados no Gráfico 1. Em ambas as situações, as proporções foram mais expressivas no sexo feminino, independente da região. Entretanto, houve variação por Macrorregionais, tanto no sexo masculino quanto no feminino, e nos dois pontos de corte de idade considerados. O percentual mais expressivo de mulheres edêntulas de 45 anos ou mais foi na Macrorregião Nordeste (39,5\%), enquanto naquelas de 60 anos ou mais foi mais elevado em Jequitinhonha (57,7\%). Quanto aos homens de 


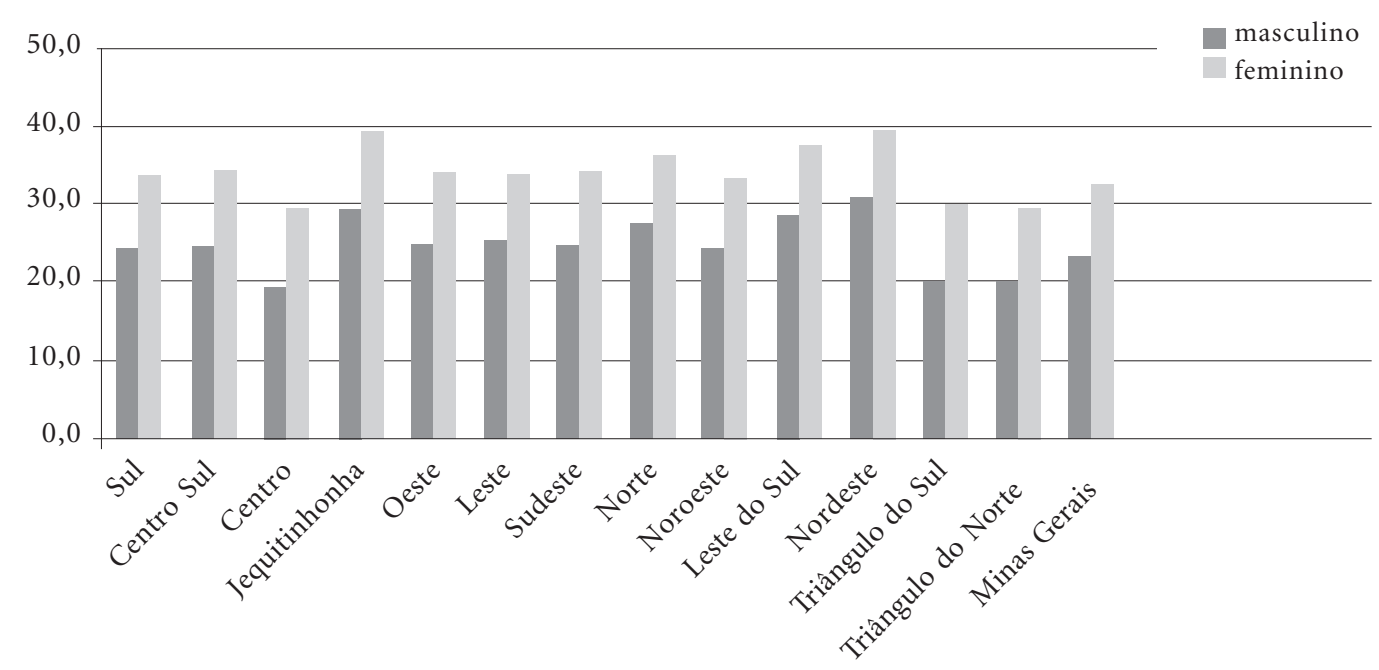

1b) 60 anos e mais

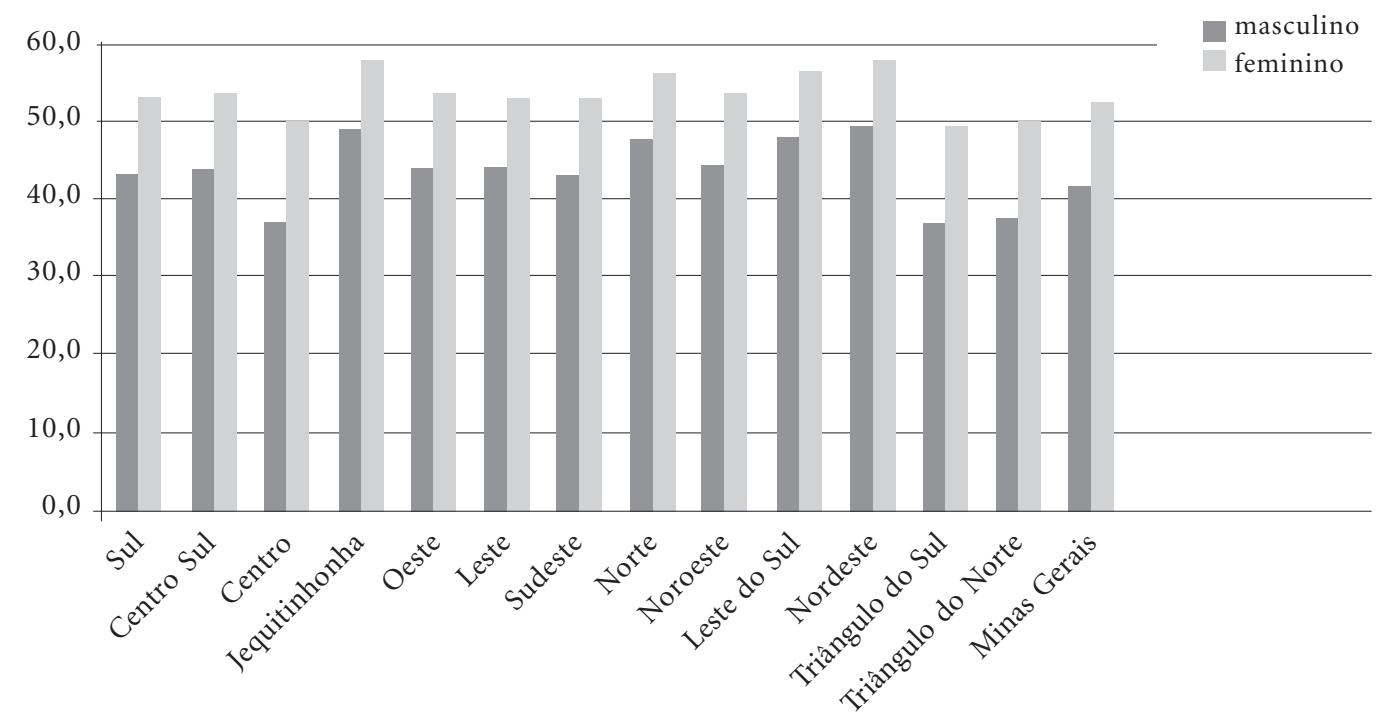

Gráfico 1. Distribuição da prevalência de edentulismo nos indivíduos de 45 anos e mais e de 60 anos e mais, segundo Macrorregionais de Saúde e sexo. Minas Gerais, 2004-2006.

Fonte: Núcleo de Pesquisa em Métodos Aplicados aos Estudos de Carga Global de Doença, Escola Nacional de Saúde Pública Sergio Arouca, Fundação Oswaldo Cruz.

45 anos ou mais, a proporção de edentulismo foi maior na Macrorregião Nordeste (30,9\%), e naqueles de 60 anos ou mais, na Macrorregião Leste do Sul (47,8\%). Nas Macrorregiões Centro, Triângulo do Norte e Triângulo do Sul, prevaleceram menores proporções de população edêntula em ambos os sexos e em ambas faixas etárias.

A Tabela 2 apresenta o número e a distribuição proporcional da carga de morbidade segun- do Grandes Grupos de causas e sexo. De forma geral, o Grande Grupo I foi responsável por $11,4 \%$ dos anos de vida vividos com incapacidade (YLD) para Minas Gerais, o Grande Grupo II por $87,2 \%$, e o Grande Grupo III por 1,4\% do total da carga de morbidade em Minas Gerais no período 2004 - 2006.

Ainda em 2004-2006, foram estimados para cárie dentária 4.489 YLD em Minas Gerais, cor- 
respondendo a 10,8\% do total de YLD estimados para todo o grupo das doenças orais, sendo $13,7 \%$ para o sexo masculino e $8,9 \%$ para o feminino. Para doença periodontal, foram estimados 3.217 YLD, gerando 7,7\% do total da doenças orais, com 9,4\% no sexo masculino e $6,6 \%$ no feminino. O edentulismo participou com 34.035 YLD, perfazendo $81,5 \%$ do total. Entretanto, $84,5 \%$ deste agravo foram atribuídos ao grupo feminino, ao passo que $76,9 \%$ ao sexo masculino.

A Tabela 3 apresenta a distribuição das taxas dos anos de vida vividos com incapacidade (YLD) de cárie dentária, doença periodontal e edentulismo, por sexo e faixa etária. As taxas de YLD de cárie dentária foram mais expressivas na faixa etária de 15 a 44 anos (em torno de 30 casos por 100.000 habitantes-ano para ambos os sexos) com valores decrescentes a partir de 45 anos.

No edentulismo, as taxas de YLD de ambos os sexos foram crescentes por faixa etária até 60 a 69 anos, cujos valores alcançaram 1.052 casos nos homens e 1.382 nas mulheres, com decréscimo para os indivíduos a partir de 70 anos. No que tange à doença periodontal, observou-se semelhanças para ambos os sexos na distribuição da taxa de YLD, crescente até a faixa etária de 60 a 69 anos.

A Tabela 4 apresenta as taxas brutas e as razões de taxas de YLD em relação à taxa global do Estado de Minas Gerais para cárie dentária, doença periodontal e edentulismo, segundo Macrorregionais de Saúde e sexo. No tocante à cárie, as disparidades das taxas em relação ao Estado foram menores, para ambos os sexos, quando comparadas ao edentulismo. No entanto, o padrão de distribuição entre as Macrorregionais de Saúde foi similar no que diz respeito às taxas superiores ao valor obtido para Minas Gerais

Tabela 3. Distribuição das taxas dos anos de vida vividos com incapacidade (YLD) de cárie dentária, doença periodontal e edentulismo, e segundo faixa etária e sexo. Minas Gerais, 2004-2006.

\begin{tabular}{crrr}
$\begin{array}{c}\text { Sexo/Faixa } \\
\text { Etária }\end{array}$ & $\begin{array}{c}\text { Cárie } \\
\text { dentária }\end{array}$ & $\begin{array}{c}\text { Doença } \\
\text { Periodontal }\end{array}$ & Edentulismo \\
\hline Masculino & & & \\
$<1$ & 0,0 & 0,0 & 0,0 \\
$1-6$ & 4,5 & 0,0 & 0,0 \\
$7-14$ & 19,5 & 0,0 & 0,0 \\
$15-29$ & 30,5 & 10,7 & 23,5 \\
$30-44$ & 31,5 & 22,2 & 59,8 \\
$45-59$ & 29,8 & 33,9 & 466,4 \\
$60-69$ & 8,3 & 54,4 & $1.052,0$ \\
$70-79$ & 4,5 & 43,9 & 16,3 \\
$80+$ & 1,8 & 29,9 & 0,0 \\
Feminino & & & \\
$<1$ & 0,0 & 0,0 & 0,0 \\
$1-6$ & 4,5 & 0,0 & 0,0 \\
$7-14$ & 19,5 & 0,0 & 0,0 \\
$15-29$ & 30,5 & 10,8 & 49,4 \\
$30-44$ & 31,5 & 22,3 & 121,5 \\
$45-59$ & 29,8 & 34,6 & 798,6 \\
$60-69$ & 8,3 & 56,6 & $1.382,2$ \\
$70-79$ & 4,5 & 46,0 & 21,4 \\
$80+$ & 1,8 & 31,3 & 0,0 \\
\hline
\end{tabular}

Fonte: Núcleo de Pesquisa em Métodos Aplicados aos Estudos de Carga Global de Doença, Escola Nacional de Saúde Pública Sergio Arouca, Fundação Oswaldo Cruz.

Tabela 2. Número absoluto e distribuição proporcional dos anos de vida vividos com incapacidade (YLD) segundo grandes grupos de causas e grupo de condições orais e segundo sexo. Minas Gerais, 2004-2006.

\begin{tabular}{|c|c|c|c|c|c|c|}
\hline \multirow{2}{*}{ Grupos de causas } & \multicolumn{2}{|c|}{ Masculino } & \multicolumn{2}{|c|}{ Feminino } & \multicolumn{2}{|c|}{ Total } \\
\hline & Número & $\%$ & Número & $\%$ & Número & $\%$ \\
\hline $\begin{array}{l}\text { Grupo I - Infecciosas e Parasitárias, } \\
\text { Maternas, Perinatais e Nutricionais }\end{array}$ & 88.185 & 12,7 & 95.763 & 10,4 & 183.948 & 11,4 \\
\hline Grupo II - Não Transmissíveis & 587.462 & 84,9 & 815.314 & 88,9 & 1.402 .776 & 87,2 \\
\hline Grupo III - Causas Externas & 16.180 & 2,3 & 6.482 & 0,7 & 22.662 & 1,4 \\
\hline Grupo II - Orais & 16.249 & 100,0 & 25.492 & 100 & 41.741 & 100 \\
\hline Cárie & 2.226 & 13,7 & 2.263 & 8,9 & 4.489 & 10,8 \\
\hline Periodontite & 1.534 & 9,4 & 1.683 & 6,6 & 3.217 & 7,7 \\
\hline Edentulismo & 12.489 & 76,9 & 21.546 & 84,5 & 34.035 & 81,5 \\
\hline Subtotal & 691.827 & 100,0 & 917.559 & 100,0 & 1.609 .386 & 100,0 \\
\hline
\end{tabular}

Fonte: Núcleo de Pesquisa em Métodos Aplicados aos Estudos de Carga Global de Doença, Escola Nacional de Saúde Pública Sergio Arouca, Fundação Oswaldo Cruz. 
Tabela 4. Taxas dos anos de vida vividos com incapacidade (YLD)* de cárie, doença periodontal e edentulismo e razão de taxa padronizada, segundo Macrorregionais de Saúde e por sexo. Minas Gerais, 2004-2006.

\begin{tabular}{|c|c|c|c|c|c|c|}
\hline \multirow[b]{2}{*}{ Macrorregionais/Sexo } & \multicolumn{2}{|c|}{ Cárie } & \multicolumn{2}{|c|}{ Doença Periodontal } & \multicolumn{2}{|c|}{ Edentulismo } \\
\hline & Taxa & $\mathbf{R T P}^{* *}$ & Taxa & $\mathbf{R T P}^{* *}$ & Taxa & RTP $^{* *}$ \\
\hline \multicolumn{7}{|l|}{ Masculino } \\
\hline Sul & 23 & 98 & 17 & 101 & 142 & 105 \\
\hline Centro Sul & 23 & 100 & 17 & 100 & 141 & 105 \\
\hline Centro & 24 & 103 & 17 & 100 & 115 & 86 \\
\hline Jequitinhonha & 24 & 102 & 16 & 98 & 171 & 127 \\
\hline Oeste & 23 & 99 & 17 & 101 & 146 & 109 \\
\hline Leste & 23 & 99 & 16 & 100 & 146 & 108 \\
\hline Sudeste & 24 & 104 & 17 & 100 & 136 & 101 \\
\hline Norte & 24 & 102 & 16 & 98 & 164 & 122 \\
\hline Noroeste & 23 & 97 & 17 & 100 & 151 & 112 \\
\hline Leste do Sul & 23 & 100 & 17 & 100 & 164 & 122 \\
\hline Nordeste & 24 & 101 & 16 & 98 & 164 & 122 \\
\hline Triângulo do Sul & 21 & 92 & 17 & 100 & 111 & 82 \\
\hline Triângulo do Norte & 23 & 97 & 17 & 101 & 118 & 87 \\
\hline Minas Gerais & 23 & 100 & 17 & 100 & 134 & 100 \\
\hline \multicolumn{7}{|l|}{ Feminino } \\
\hline Sul & 23 & 98 & 17 & 100 & 220 & 102 \\
\hline Centro Sul & 23 & 100 & 17 & 100 & 219 & 102 \\
\hline Centro & 24 & 103 & 17 & 101 & 200 & 93 \\
\hline Jequitinhonha & 24 & 102 & 16 & 97 & 255 & 118 \\
\hline Oeste & 23 & 99 & 17 & 101 & 225 & 105 \\
\hline Leste & 23 & 99 & 17 & 100 & 224 & 104 \\
\hline Sudeste & 24 & 104 & 17 & 100 & 217 & 101 \\
\hline Norte & 24 & 102 & 17 & 98 & 246 & 114 \\
\hline Noroeste & 23 & 97 & 17 & 100 & 230 & 107 \\
\hline Leste do Sul & 23 & 100 & 17 & 100 & 248 & 115 \\
\hline Nordeste & 24 & 101 & 17 & 98 & 253 & 117 \\
\hline Triângulo do Sul & 21 & 92 & 17 & 100 & 190 & 88 \\
\hline Triângulo do Norte & 23 & 97 & 17 & 101 & 198 & 92 \\
\hline Minas Gerais & 23 & 100 & 17 & 100 & 215 & 100 \\
\hline
\end{tabular}

* por 100.000 mil habitantes (2) Razão de taxa padronizada

Fonte: Núcleo de Pesquisa em Métodos Aplicados aos Estudos de Carga Global de Doença, Escola Nacional de Saúde Pública Sergio Arouca, Fundação Oswaldo Cruz.

como um todo para ambos os sexos. Em contrapartida, nas Macrorregionais Sul, Oeste, Leste, Noroeste, Triângulo do Norte e Triângulo do Sul, as taxas foram inferiores ao Estado como um todo: $98 \%, 99 \%, 97 \%, 92 \%$ e $97 \%$, respectivamente, para ambos os sexos.

Não foram observadas grandes variações na distribuição das taxas padronizadas de doença periodontal para ambos os sexos. Em relação ao edentulismo, observou-se que as Macrorregiões de Saúde Jequitinhonha, Norte de Minas, Leste do Sul e Nordeste alcançaram as maiores taxas do YLD por 100.000 habitantes para o sexo masculino, resultando em taxas padronizadas de $22 \%$ a $27 \%$ superiores à taxa de carga global de doença de Minas Gerais como um todo. Entretanto, no sexo feminino, estas macrorregiões atingiram taxas padronizadas superiores às do Estado, de $14 \%$ a $18 \%$. Todavia, os percentuais foram sempre inferiores ao do sexo masculino. No outro extremo, nas Macrorregionais Centro, Triângulo do Norte e Triângulo do Sul, as taxas padronizadas foram inferiores às de Minas Gerais como um todo, respectivamente: $86 \%, 87 \%$ e $82 \%$ para homens e $93 \%$, 92\% e $88 \%$ para mulheres.

\section{Discussão}

A estimativa da carga global de doença de cárie dentária, doença periodontal e edentulismo para o Estado de Minas Gerais, no período 2004 a 
2006, evidenciou a ocorrência destas doenças em praticamente todas as faixas etárias e afetaram, sobremaneira, o sexo feminino.

O número de YLD estimados para as condições orais foi de 41.741, com percentual participativo no total da carga de morbidade do Estado de Minas Gerais na ordem de 2,9\%. No Brasil, resultados do Estudo de Carga Global de Doença para o ano de $1998^{12}$ evidenciaram que $2,4 \%$ do total de YLD corresponderam às condições orais.

No tocante ao componente de morbidade, foram estimados, para cárie dentária, 4.489 YLD, com participação relativa de $10,8 \%$ no total das doenças orais e $0,3 \%$ no total da morbidade. Outros estudos sobre carga global de doença estimaram percentuais para cárie dentária que variaram de $0,9 \%$ a $1,1 \%{ }^{20,21}$.

No tocante à contribuição de cada agravo oral à carga global de doença, os achados do presente estudo foram diferenciados aos encontrados em outros com temática semelhante. Enquanto alguns trabalhos apontam a cárie dentária como a condição oral com participação percentual mais relevante $^{20-22}$, este estudo estimou que o edentulismo foi o agravo mais contributivo para a carga global de doenças orais no Estado de Minas Gerais.

A participação relativa do edentulismo no cômputo das doenças orais foi de $81,5 \%$ e no total de morbidade foi de 2,1\%, com estimativa de 34.035 YLD. A estimativa, para a região das Américas em 2004, foi de 0,3\% do total da carga global de doença ${ }^{11}$ e outros estudos apontam estimativas de edentulismo na ordem de $0,3 \%$ a $0,4 \%$ do total de carga de morbidade ${ }^{21,22}$. Na Austrália, em 2003, foram estimados 5.264 YLD, perfazendo uma participação relativa de $0,3 \%$ ( $0,2 \%$ para o sexo masculino e $0,4 \%$ para o feminino) no total da carga de morbidade ${ }^{17}$.

O número de DALY estimados para doença periodontal no Estado de Minas Gerais foi de 3.217 , correspondendo a $0,2 \%$ do total de YLD. Em Victoria, Austrália, em 1996, cerca de 0,6\% do total de YLD foram atribuídos à doença periodontal, com estimativa $0,6 \%$ para homens e $0,7 \%$ para mulheres ${ }^{22}$. Em relação a este agravo, o estudo da Organização Mundial de Saúde de 2004, estimou 39.000 DALY, resultando em $0,01 \%$ do total de carga de doença ${ }^{11}$.

Como as estimativas de edentulismo de $\mathrm{Mi}$ nas Gerais evidenciaram uma maior participação deste agravo no cômputo do total de YLD de doenças orais supõe-se que as estimativas de Minas Gerais estariam superiores porque, no presente estudo, o peso da incapacidade, ponderado para tratamento e não tratamento dos ca- sos foi de 0,019, valor quase cinco vezes superior àquele empregado, por exemplo, no estudo de Carga Global de Doença na Austrália em 2003, por exemplo. No estudo australiano, o número de indivíduos edêntulos foi menor, mas também se considerou que $100 \%$ eram tratados e o peso estimado foi de 0,004 .

Quanto à análise de edentulismo por $\mathrm{Ma}$ crorregionais de Saúde, este estudo demonstrou que regiões menos desenvolvidas, como Jequitinhonha, Norte de Minas e Nordeste, refletem tendência de maiores prevalências para as condições orais quando comparadas às localidades de melhor nível socioeconômico. Ao longo de toda a análise, independentemente do indicador de saúde utilizado, se a prevalência ou os anos de vida vividos com incapacidade, estas regiões concentraram maiores casos de edentulismo, sempre ainda mais desfavoráveis no sexo feminino.

Todavia, especificamente no tocante à prevalência de edentulismo nos indivíduos de 45 anos e mais e nos de 60 anos e mais, observou-se que, além das regiões menos favorecidas reunirem maiores proporções de indivíduos com edentulismo e no sexo feminino, estas áreas contam com proporções um pouco mais elevadas de mulheres que se tornam edêntulas precocemente, pois as diferenças nas prevalências entre os grupos etários são menores quando comparadas às regiões de melhor nível de desenvolvimento.

Quanto menor a divergência entre os anos de vida vividos com incapacidade aos 45 anos e 60 anos de idade, pior é a situação do agravo, pois mais indivíduos desenvolvem a condição em questão em idades menos avançadas. Naturalmente, a expectativa é de que esses diferenciais sejam significativamente maiores, ou seja, que a prevalência de indivíduos edêntulos de 60 anos e mais seja expressivamente maior do que aquela nos de 45 anos e mais.

A importância do edentulismo no contexto das condições orais destaca-se no momento em que se torna consequência de alguns agravos bucais, dentre eles a cárie e a doença periodontal. Sendo a cárie uma doença infecciosa que ataca as estruturas dos dentes e a doença periodontal uma inflamação causada por infecção dos tecidos de suporte dos mesmos, estes agravos, quando não tratados têm, como consequência, a perda dentária.

Partindo-se da observação de que o edentulismo afeta mais as mulheres ${ }^{8,23,24}$, pressupõe-se que esta associação entre sexo e frequência da doença seja influenciada pelo uso de serviços de saúde. Dados da Pesquisa Nacional por Amostragem de Domicílios (PNAD) de 2003 aponta- 
ram uma chance 1,2 vezes maior de os homens nunca terem procurado serviço odontológico quando comparados a mulheres ${ }^{10}$. Este diferencial por sexo também foi encontrado em alguns estudos internacionais ${ }^{4,5}$

De outro modo, cabe ressaltar que o impacto da cárie não foi o mesmo nos diferentes momentos da evolução da sociedade humana. O desenvolvimento introduziu novos hábitos alimentares e modos de vida e, a partir do processo de urbanização, este agravo tornou-se mais prevalente $^{25}$. Salienta-se que o padrão alimentar de baixa qualidade, aliado ao consumo excessivo de açúcar, relaciona-se à presença de cárie e que uma dieta inadequada geralmente é mais previsível de acometer indivíduos de classes sociais menos favorecidas, razão pela qual cresce a chance de estes desenvolverem mais frequentemente o agravo. Aliada a esta lógica está a questão dos hábitos de higiene, fortemente associados à cárie, e o acesso a serviços odontológicos.

As condições orais fazem parte do cômputo dos agravos mais comuns e dos principais problemas de saúde a nível mundial. Grande parcela de seus efeitos relaciona-se à dor, desconforto, sequelas físicas e autoestima, ou seja, os sintomas estão fixados mais nos aspectos ligados à morbidade do que à mortalidade. Além disso, merece destaque o impacto no sistema de saúde relacionado às despesas inerentes ao tratamento.

Ponderando-se que, em sua maioria, os diagnósticos de cárie e de doença periodontal são feitos mediante exames clínicos, com o uso dos índices CPOD/CEO para o primeiro e do Índice Periodontal Comunitário (CPI) para o segundo, um maior impedimento para vigilância destes agravos seriam os altos custos e recursos necessários para coleta de dados clínicos.

Uma das limitações do presente estudo diz respeito à escassez de parâmetros locais para o cálculo da carga de doenças das condições orais de Minas Gerais e suas Macrorregionais de Saúde. As informações de prevalência e incidência de cárie, doença periodontal e edentulismo foram obtidas estimando-se, para nível local, informações de âmbito mais abrangente, principalmente nacional. Isto implicou em assumir que, mesmo sendo o Brasil um país heterogêneo, com diferenciais regionais marcantes, as estimativas possuíam comportamento semelhante ao conjunto das localidades pesquisadas nos diferentes inquéritos usados neste estudo.

Outra limitação das estimativas apresentadas para a cárie dentária refere-se à possibilidade de a carga de doença deste agravo possivelmente encontrar-se superestimada em função, principalmente, do uso das informações de assistência dental para caracterizar o agravo. Para as informações de sequelas de cárie, necessárias para estimar a carga global de doença, os casos tratados (obturados) foram definidos a partir do tratamento dental recebido no último ano do inquérito PMS $\mathrm{AB}$, que pode abarcar cuidados odontológicos mais abrangentes do que necessariamente aqueles relacionados somente à doença. O mesmo diz respeito aos casos não tratados (dentes perdidos), definidos a partir da extração de dente, que podem não contemplar somente a extração devido à cárie.

Optou-se por não utilizar os índices CPOD e CEO na estimativa de carga global de doença de cárie dentária pois, mesmo sendo a forma mais usual para quantificar o agravo, estes índices expressam cárie por número de dentes e, na metodologia dos estudos de Carga Global de Doença, as estimativas são baseadas na incidência anual por pessoa.

Algumas limitações do uso do CPOD e CEO para diagnóstico de cárie já foram apontadas na literatura, tais como: não retratam perdas do dente por doença periodontal ou razões ortodônticas, são passíveis de interpretações distintas dos estágios iniciais de cárie por parte dos examinadores, ocorrendo subestimação quando cáries de esmalte não são consideradas e não permitem identificar cárie radicular ${ }^{26}$.

O edentulismo, mesmo atingindo mais expressivamente a população adulta e idosa, não é resultado do envelhecimento populacional e sim consequência de precárias condições de saúde oral, pois foi observada, neste estudo, a presença da doença nos adultos jovens de faixa etária 15 a 29 anos. Isso pode ser explicado pela alta prevalência de cárie na população brasileira, possivelmente não tratada, e ainda reflexo das políticas de oferta de serviços odontológicos de exodontia ${ }^{27}$.

A metodologia proposta para os estudos de Carga Global de Doença estima a carga do edentulismo a partir da perda total de dentes, não abarcando estimativas de edentulismo funcional. Entretanto, o edentulismo funcional acarreta efeitos muito mais significativos devido à ausência de alguns, mas não todos os dentes, pois além de gerar danos físicos, estéticos e psicológicos, há o problema do custo para restauração, evidentemente menos acessível para indivíduos com rendimento precário.

De forma geral, as estimativas da carga global de doença das condições orais deste estudo foram robustas e comparáveis a outras pesqui- 
sas da mesma natureza. As limitações metodológicas de ausência de informação local de prevalência ou incidência dos agravos por sexo e faixa etária, para o cálculo do componente de incapacidade YLD, impelindo o uso de pressupostos para preenchimento de lacunas de informação, foram semelhantes àquelas encontradas nos outros estudos executados no mundo ${ }^{17,21}$.

Ressalta-se o caráter deste estudo ao adotar as Macrorregionais de Saúde como escala de análise geográfica. Considerando que se trata de uma divisão político-administrativa, novas análises poderão ser conduzidas a fim de reavaliar a ocorrência dos problemas orais em outros níveis de agregação.

De forma geral, os resultados evidenciaram a importância de se estimar a Carga Global de Doença das condições orais, enquanto a análise ecológica mostrou que piores condições de vida não podem ser dissociadas das doenças bucais, pois para as áreas com menor nível de desenvolvimento estimaram-se maiores taxas de YLD.

As características sociais e econômicas de uma localidade tendem a determinar, pelo menos em parte, o acesso local aos serviços de saúde oral. Contudo, ainda que a pobreza não seja determinante direta dos agravos bucais, o presente estudo aponta evidências empíricas expressas pelas maiores cargas de doença na população com baixas condições de vida.

A integração da saúde oral nas estratégias de promoção da saúde geral pode melhorar significativamente ambas, pois além de os agravos orais associarem-se com outras doenças crônicas, um e outro possuem geralmente fatores de risco em comum. Torna-se igualmente relevante a disponibilização de dados, mais especificamente, registros de morbidade que permitam prover informações que auxiliem na formulação de políticas públicas, não somente para o diagnóstico e tratamento, mas, de forma prioritária, para a prevenção.

A contribuição ao DALY dos agravos orais foi predominantemente no componente de incapacidade YLD. A integração de desfechos fatais e não fatais na metodologia de estimação para a Carga Global de Doença, ressalta sua importância no diagnóstico de saúde de uma população. Por outro lado, um padrão de saúde retratado por indicadores de mortalidade, em sua natureza mais acessíveis devido à disponibilidade dos sistemas de informação brasileiros de domínio público, não revelaria o peso das morbidades. As doenças que participam quase que exclusivamente do quadro de morbidade, como as orais, incapacitantes do ponto de vista da qualidade de vida dos indivíduos, não participariam do perfil de saúde populacional, se apenas a mortalidade fosse computada para compor as estimativas de doenças que mais grassam nos indivíduos.

Tendo em vista que os estudos de Carga Global de Doença utilizam o indicador DALY como uma medida sumária para eventos fatais e não fatais combinados, as aplicações no campo da Saúde Pública deste indicador assemelham-se à utilização comum de outros, que resumem a situação de saúde de uma população, podendo ser utilizados também para avaliação de mudanças ou tendências durante um período de tempo.

As doenças orais são um fenômeno complexo, multifacetado, com variadas determinações que se interligam e se interagem. Entretanto, o padrão de utilização da assistência em saúde oral é reflexo das oportunidades de consumo de serviços nos diferentes segmentos sociais, mostrando que esses serviços continuam sendo um produto desigualmente distribuído e essas desigualdades são, também, moldadas por fatores socioeconômicos.

Ainda que as diretrizes da política de atenção à saúde oral estabeleçam a atenção básica como componente principal na reestruturação da assistência, utilizando os recursos humanos das Equipes de Saúde Família, é essencial a ampliação e a qualificação constantes do acesso aos serviços, garantindo a incorporação da atenção integral à saúde bucal.

\section{Colaboradores}

JC Mota participou da redação do artigo, análise e interpretação dos dados. IC Leite, JG Valente e JMA Schramm participaram da análise e interpretação dos dados.

\section{Agradecimentos}

Agradecemos à Secretaria de Estado de Saúde de Minas Gerais pelo financiamento deste estudo, fundamental para a estimativa da Carga de Doenças de Minas Gerais. 


\section{Referências}

1. Fernandes LS, Peres MA. Associação entre atenção básica em saúde bucal e indicadores socioeconômicos municipais. Rev Saude Publica 2005; 39(6):930936.

2. Caldas Júnior AF, Caldas KU, Oliveira MRM, Amorim AA, Barros PMF. O Impacto do edentulismo na qualidade de vida de idosos. Rev Cienc Med (Campinas) 2005; 14(3):229-238.

3. Mignogna MD, Fedele S. The Neglected Global Burden of Chronic Oral Diseases. J Dent Res 2006; 85(5):390-391.

4. Müller F, Naharro M, Carlsson GE. What are the prevalence and incidence of tooth loss in the adult and elderly population in Europe? Clin Oral Implants Res 2007; 18(Supl. 3):2-14.

5. Australian Research Centre for Population Oral Health. The University of Adelaide, South Australia. Decline of the edentulism epidemic in Australia. Aust Dent J 2007; 52(2):154-156.

6. Narvai PC, Frazão P, Roncalli AG, Antunes JLF. Cárie dentária no Brasil: declínio, polarização, iniqüidade e exclusão social. Rev Panam Salud Publica 2006; 19(6):385-393.

7. Boyce WT, Den Besten PK, Stamperdahl J, Zhan L Jiang Y, Adler NE, Featherstone JD. Social inequalities in childhood dental caries: The convergent roles of stress, bacteria and disadvantage. Soc Sci Med 2010; 71(9):1644-1652.

8. Barbato PR, Peres MA. Perdas dentárias em adolescentes brasileiros e fatores associados: estudo de base populacional. Rev Saude Publica 2009; 43(1):13-25.

9. Baldani MH, Vasconcelos AGG, Antunes JLF. Associação do índice CPO-D com indicadores sócioeconômicos e de provisão de serviços odontológicos no Estado do Paraná, Brasil. Cad Saude Publica 2004; 20(1):143-152.

10. Pinheiro RS, Torres TZG. Uso de serviços odontológicos entre os Estados do Brasil. Cien Saude Colet 2006; 11(4):999-1010.

11. World Health Organization (WHO). The global burden of disease: 2004 update. [Internet]. Geneva: 2008. [acessado 2014 maio 22]. Disponível em: http:// www.who.int/healthinfo/global_burden_disease/ 2004_report_update/en/

12. Schramm JMA, Oliveira AF, Leite IC, Valente JG, Gadelha AMJ, Portela MC, Campos MR. Transição epidemiológica e o estudo de carga de doença no Brasil. Cien Saude Colet 2004; 9(4):897-908.

13. Leite IC, Costa MFS, Valente JG, Schramm JMA, Daumas RP, Rodrigues RN. Carga de Mortalidade Estado de Minas Gerais, 2005. Belo Horizonte: Secretaria de Estado da Saúde de Minas Gerais/CGDANT; 2009. (Relatório técnico, 2009).

14. Szwarcwald CL, Viacava F. Pesquisa Mundial de Saúde: aspectos metodológicos e articulação com a Organização Mundial da Saúde. Rev Bras Epidemiol 2008; 11(Supl. 1):58-66.

15. Brasil. Ministério da Saúde (MS). Secretaria de Atenç̃o à Saúde. Departamento de Atenção Básica. Projeto SB Brasil 2003: Condições de saúde bucal da população brasileira, principais resultados. Brasília: MS; 2004.
16. Murray CJL, Lopez AD. Global Health Statistics: A Compendium of Incidence, Prevalence and Mortality Estimates for over 200 conditions. Geneva: World Health Organization (WHO); 1996.

17. Begg S, Vos T, Barker B, Stevenson C, Stanley L, Lopez AD. The burden of disease and injury in Australia 2003. Canberra: AIHW; 2007. PHE 82.

18. World Health Organization (WHO). DISMOD II. Software and manual. Geneva: WHO; 2001.

19. Murray CJL, Lopez AD. The Global Burden of Disease: a comprehensive assessment of mortality and disability from diseases, injuries, and risk factors in 1990 and projected to 2020. Cambridge: Harvard School of Public Health; 1996.

20. Australia. Victoria. Epidemiology Section, Health Intelligence and Disease Control, Public Health and Development Division,Victorian Government Department of Human Services. Victorian Burden of Disease Study [Internet]. Melbourne, Victoria: 2001. [acessado 2014 maio 21]. Disponível em: http:// www.health.vic.gov.au/healthstatus/composite/bod/ bod-vic.htm

21. Pike A, Baade P, Harper C, Muller S, Kennedy B. Quantifying the burden of disease and injury in Queensland 1996-1998. Information circular 61. Brisbane: Health Information Centre, Queensland Health; 2002.

22. Australia. Victoria. Epidemiology Section, Health Intelligence and Disease Control, Public Health and Development Division, Victorian Government Department of Human Services. Victorian Burden of Disease Study [Internet]. Melbourne, Victoria: 1999. [acessado 2014 maio 21]. Disponível em: http:// www.health.vic.gov.au/healthstatus/composite/bod/ bod-vic.htm

23. Barbato PR, Nagano HCM, Zanchet FN, Boing AF, Peres MA. Perdas dentárias e fatores sociais, demográficos e de serviços associados em adultos brasileiros: uma análise dos dados do Estudo Epidemiológico Nacional (Projeto SB Brasil 2002-2003). Cad Saude Publica 2007; 23(8):1803-1814.

24. Moreira RS. Perda dentária em adultos e idosos no Brasil: A influência de aspectos individuais, contextuais e geográficos [tese]. São Paulo: Universidade de São Paulo; 2009.

25. Gomes D, Da Ros MA. A etiologia da cárie no estilo de pensamento da ciência odontológica. Cien Saude Colet 2008; 13(3):1081-1090.

26. Pinto VG. Saúde Bucal Coletiva. $4^{\mathrm{a}}$ ed. São Paulo: Livraria Editora Santos; 2000.

27. Baldani MH, Almeida ES, Antunes JLF. Equidade e provisão de serviços públicos odontológicos no estado do Paraná. Rev Saude Publica 2009; 43(3):446454

Artigo apresentado em 12/06/2013

Aprovado em 30/09/2013

Versão final apresentada em 14/10/2013 